\title{
INTEGRATION OF STRATEGIC AND OPERATIONAL ASSET MANAGEMENT
}

\author{
Andreas STEFFEN \\ Christian WEMHOFF \\ Sven HÜBNER \\ ENERVIE AssetNetWork -Germany ENERVIE AssetNetWork - Germany entellgenio - Germany entellgenio - Germany \\ Heiko SPITZER \\ andreas.steffen@enervie-assetnetwork.de Christian.wemhoff@enervie-assetnetwork.de sven.huebner@entellgenio.com heiko.spitzer@entellgenio.com
}

\begin{abstract}
Coordination of strategic and operational asset management at ENERVIE AssetNetWork was a challenge in the past. ENERVIE AssetNetWork developed and implemented a practical and simple solution supported by two software tools. These simple tools improve the coordination between sustainable strategic asset planning and the tactical operative planning by:

1. an automated provision of required data directly from asset service's storage systems within a few hours

2. "real-time" support of commissioning through automated comparison of strategic and operative projects

The chosen approach has been implemented successfully within one year and shows first benefits.
\end{abstract}

\section{CHALLENGE AND OBJECTIVE}

ENERVIE provides energy services for electricity, gas and water in Germany. ENERVIE's subsidiary, Enervie AssetNetWork, operates distribution grids for electricity, gas and water in Hagen, Lüdenscheid and the surrounding cities. In the past, Asset Management and Asset Service at ENERVIE AssetNetWork faced the challenge to capture the technical long-term asset development and the operational short-term project planning within one planning process supported by the appropriate IT-tools. However, only this approach guarantees the stringent implementation of strategic objectives (Asset Management) as well as the operational project planning (Asset Service) to have grid budget, quality and risks aligned.

\section{Asset Service}
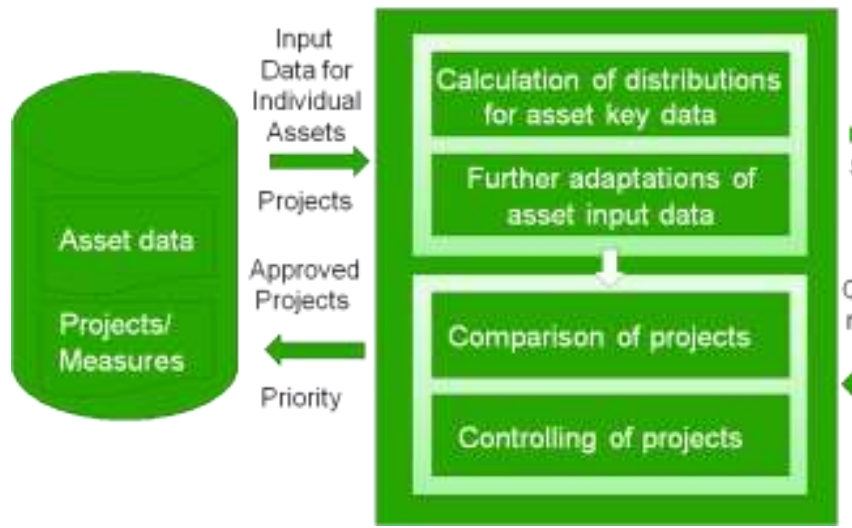

The strategic technical Asset Planning is based on the validation of asset groups, while the operational Asset Planning is driven by measures/projects, which leads bottom-line to different results.

Based on these facts, ENERVIE AssetNetWork developed and implemented a practical and simple approach aiming to improve the iterative adjustment process between Asset Management and Asset Service (Figure 1). The result is an aligned objective measure/project planning and an aligned integrated asset strategy (medium/long-term) for the electricity, gas and water grid. Both objectives, strategic as well as operative targets of Enervie AssetNetWork are aligned and fulfilled. This approach - based and supported by two simple software tools - is already successfully applied in the daily work processes of Asset Management and Asset Service.

\section{ASSET MANUAL AND SAME DATA BASE}

Asset Service at ENERVIE is responsible for the operational planning of individual projects/measures and supervises the current asset states for the electricity, gas and water grid.

Asset Management at ENERVIE AssetNetWork is responsible for the strategic planning of the electricity, gas and water grid and focuses on the overall maintenance and investment strategies and oversees the allocation of financial resources. Asset Management further ensures the commissioning of overall projects/measures in line with the two main strategic targets of the company: conservation of grid substance and stable profitability.

\section{Asset Management}

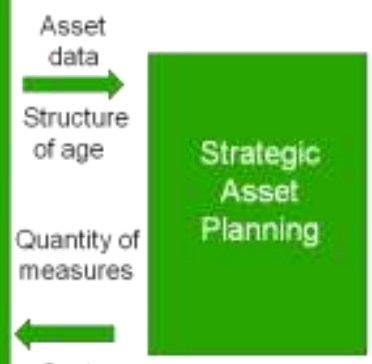

Costs

Asset

Planning

Figure 1: Interface between Asset Management and Asset Service 
To support Asset Management, ENERVIE AssetNetWork relies on asset strategy simulation and optimization (ASP). The Asset Simulation/Optimization is provided by an industry proofed software tool $[1,2,3,4]$ which is based on the transparent method of cause and effect dependencies $[5,6]$

The so-called Asset Manual builds the basis of a transparent, contemporary and advanced communication between Asset Management and Asset Service. The manual contains a description of all relevant data for the determination of the states of asset types and their correlation. Thus, the asset manual is required and crucial for the automation of the interface between Asset Management and Asset Service.

The current asset states of individual asset groups are calculated or directly imported from the different source systems. The results are automatically written back to the source systems and subsequently they are made available and provided to the strategic asset planning in an aggregated format. Thereby, an identical database (DBViews) is being established with regard to the asset states for the particular planning in Asset Service on the one hand and for the Asset Management on the other hand. This enables the support of an objective comparison of projects/measures between Asset Management and Asset Service.

\section{AUTOMATED PROVISION OF REQUIRED DATA DIRECTLY FROM ASSET SERVICE'S STORAGE SYSTEMS}

The strategic asset management simulation/optimization requires various data from Asset Service and other sources within ENERVIE AssetNetWork. The former Excel-import and manual data preprocessing is replaced by an automated interface to load and aggregate data from various database sources and software tools such as NIS/GIS (Figure 2). This data processing tool helps to reduce the time and effort to prepare the needed data for the requirements of the strategic asset management simulation/optimization significantly.

The data processing tool is connected to all important database sources within ENERVIE AssetNetWork. Data from the Asset Service such as stock data, aging parameters and data from other parts of ENERVIE such as financial and cost parameters as well as information on actual and planned projects/measures are loaded into the data processing tool.

On the one hand, the Data processing tool aggregates and links data from all these sources and derives the required parameters for the asset strategy simulation and optimization. The tool provides standardized procedures that can be applied whenever the basic data have changed. The interface allows monitoring the quality of the data and indicates if data is missing. Furthermore, it is possible to change the specification of the asset states on the front-end of the data processing tool, to recalculate the asset states and to evaluate the impact of the changes online.

On the other hand, the data processing tool provides automated data processing back to the connected data sources. The interface translates the results of ASP simulations into target parameters for the Asset Service and other parts of ENERVIE AssetNetWork.

Using the data processing tool provides various benefits: First of all, using the interface is faster than processing Excel-sheets manually. For the large amount of data required and mathematical steps to be taken, manual data processing via Excel is a very complex task.

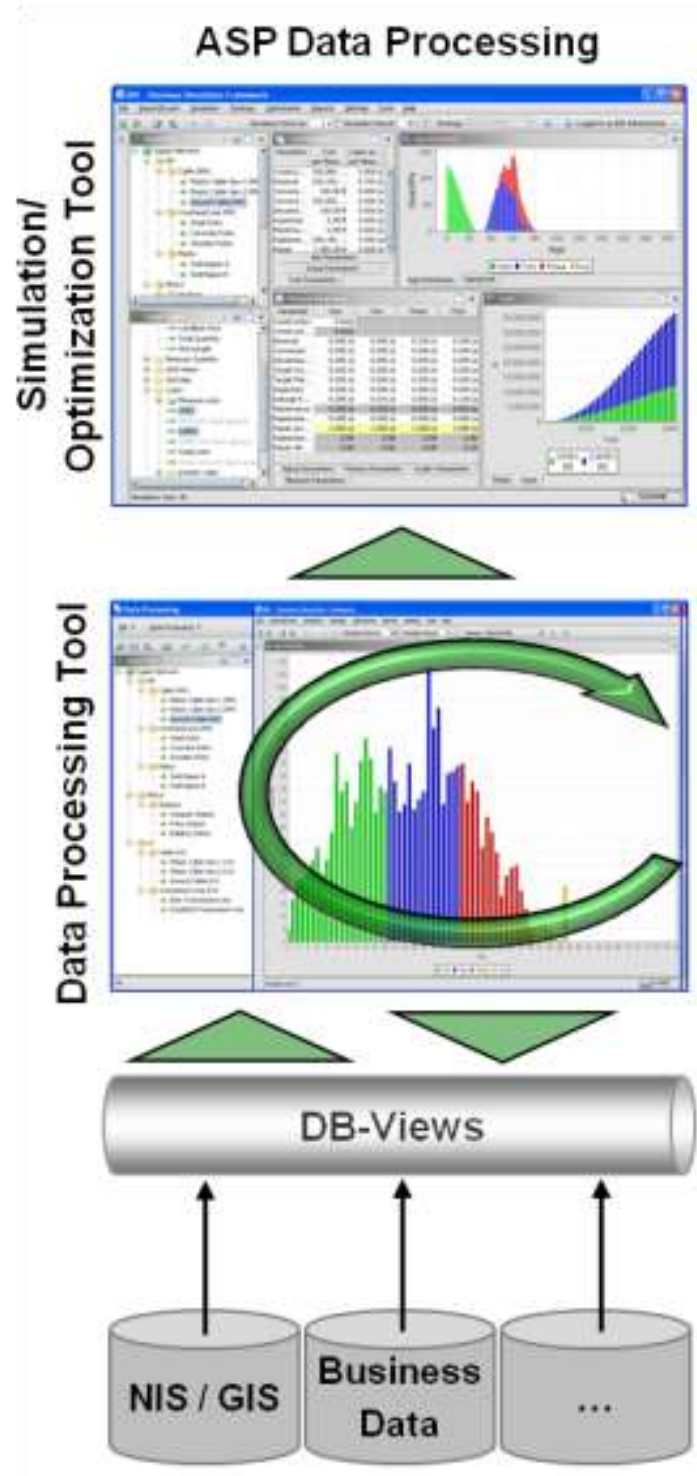

Figure 2: Data processing tool 
Secondly, the interface to increase the quality of asset data by providing a standard procedure is revised and approved from all parties. Further the process ensures that all data are up to date and match with the current information in different parts of ENERVIE.

Thirdly, the interface provides a comprehensible documentation of all steps during data processing. The asset data mapping, aggregation and processing can easily be reproduced every year (or month) with little effort. Changes to the process can be easily implemented and monitored.

All in all the automated asset data processing interface facilitates the processing of the asset data significantly. At ENERVIE AssetNetWork the annual setup for the asset strategy simulation and optimization will be done in an automated and comprehensible way within a very short timeframe and with a very high quality.

\section{COMPARISON OF PROJECTS FROM ASSET MANAGEMENT AND ASSET SERVICE}

Through a structured interface, the results of the bottomup planning in Asset Service and the top-down planning in Asset Management are imported into a "project alignment tool" for comparison and evaluation of the planned projects. By using this additional and separate software tool for comparison of planned projects the adaption of the existing planning tools of Asset Management and Asset Service is avoided.

Asset Service provides information on the operative projects currently conducted and planned. This fairly detailed bottom-up information lists projects e.g. for individual streets and provides relevant asset data for each project, such as quantity and state of assets affected, current status of the project, costs, importance etc.

The project alignment tool then maps this information with top-down objectives defined by the Asset Management. The key figures required for the mapping are imported directly from the asset simulation and optimization tool (Figure 3). This approach allows a comparison of key figures (e.g. investments, asset level by effort, and quantities replaced by each measure) for top-down and bottom-up planned projects.

As a result, the project alignment tool enables an efficient and effective alignment between strategic and operational planning. Also a transparency for both, the Asset Service and the Asset Management, is achieved. The project alignment tool provides a kind of "market place", thus a platform where operative and strategic planning approaches can be fine-tuned and brought into agreement.

\section{USING THE PROJECT ALIGNMENT TOOL FOR COMPARISON OF PROJECTS}

The project alignment tool builds the bridge between strategic Asset management and Asset service: it is assured that the asset strategy will be implemented. Within the tool individual tasks can be prioritized and approved (Figure 3). The project alignment tool shows differences between Asset Management and Asset Service through a simple "traffic light" display. The Asset Management can then approve each project/ measure from the list of all currently planned operative projects/measures. The tool shows, how the approved measures would affect the overall CAPEX/OPEX expenditures. If the list of projects/measures does not meet all budget constraints, Asset Management and Asset Service reiterate to find a common and agreed solution.

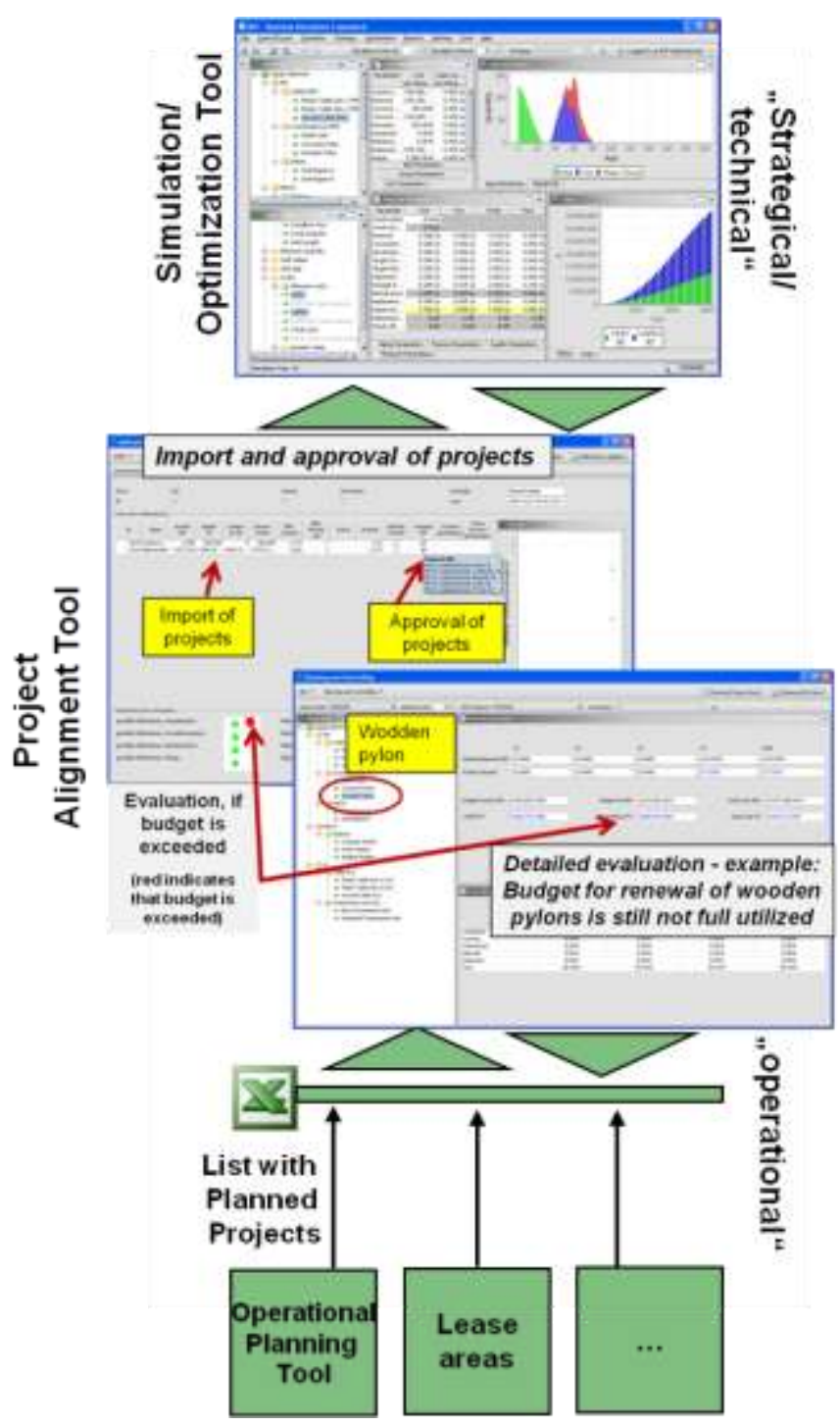

Figure 3: Project alignment tool 
Only a structured and constructive discussion between the partners in Asset Management and Asset Service can solve differences in planning. Differences in planning will lead to an adaption of the asset strategy and/or of the operational planning.

Reasons for differences in planning may be positioned in Asset Management and/or Asset Service. Perhaps there are local needs (e.g. investments programs of cities) to invest more in certain areas which was not taken into account or known when the asset strategy was developed. Then, the asset manager has to adapt the current asset strategy. But it might also be that in Asset Service the chosen asset strategy is simply not reflected in the planned projects. Then Asset Service has to change its planning to get the projects aligned with the chosen asset strategy (see Figure 4).

\section{Budget Allocation \& Risk Impact - Optimized}

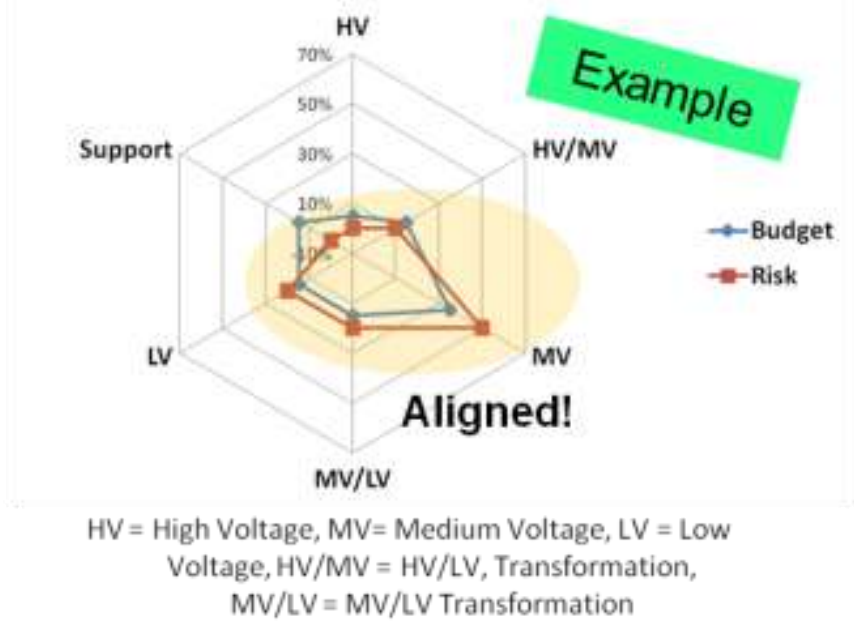

Figure 4: Aligned budget allocation

In both cases the staff from Asset Service and Asset Management have to discuss in detail what the reason is and what has to be done to fix the difference. After one or more iterations, there will be a mutual approval of the planned projects.

All in all, the "project alignment tool for comparison of planned projects" outlines the implications of the individual projects on the overall asset strategy. The project alignment tool provides the platform for constructive discussions between asset management and Asset service and thus leads to a more realistic planning in line with constraints from finance or regulation.

The tool further provides a standardized management process leading to an integrated implementation of the projects in line with the technical and strategic requirements. In addition, the project alignment tool helps to increase the mutual understanding between people from Asset Service and Asset Management.

\section{BENEFITS/OUTLOOK OF THE APPROACH}

The implementation of the described approach (which took around a year) to coordinate sustainable strategic asset planning and the tactical operative planning has proved to be successful in daily work of ENERVIE AssetNetWork since 2012. Besides some process changes, two software tools were developed which also fostered the discussions between Asset Management and Asset Service. To define a standardized process to coordinate operative and strategic asset planning, a lot of constructive discussions were necessary. In addition the data processing tool and project alignment tool create benefits by reducing time and effort and at the same time increasing quality of work. For example: the time to fill the ASP-tool with the required data has been reduced from around 6 weeks to a few hours.

It was important that the data processing tool and project alignment tool did not require fundamental adjustments of the existing software tools for asset planning. The two new software tools were fully integrated to them.

The constructive discussions between Asset Management and Asset Service (which are now possible on project level with a "real-time" information support) lead to an objective asset planning and implementation of necessary projects in the grid for the welfare and benefit of ENERVIE AssetNetWork (see Figure 4). Additionally, the mutual understanding and acceptance between the individual partners in Asset Service and Asset Management has improved considerably. Based on the current success of the approach further extensions and increments are planned.

\section{REFERENCES}

[1] Kallweit, T.; Schwarz, U.; Spitzer, H.: „Asset Strategy Planning - Simulation als Basis fundierter und nachhaltiger Asset Strategien“, in: et Energiewirtschaftliche Tagesfragen, 55. Jg. (2005), Heft 7, S. 488-489;

[2] Gaul, A. J.; Spitzer, H.: „Asset Simulation - an approach to predict the long term monetary consequences of maintenance and renewal strategies for electrical grids", CIRED, 19th International Conference on Electricity Distribution, Wien, 21.24.5.2007, Paper 0668.

[3] Dutz, A., Spitzer, H., Schulterschluss zwischen Techniker und Kaufmann, et Energiewirtschaftliche Tagesfragen 59. Jg. (2009) Heft 4

[4] Spitzer, H., Engels, C.: „Dynamic Asset Simulation - Risk Management am Beispiel der Energieversorgung“, Riskconf, München, 2009.

[5] Forrester, J.W. 1961, Industrial Dynamics, OR, Productivity Press.

[6] Sterman, J.D., 2000, Business Dynamics, Systems Thinking and Modeling for a Complex World, Boston, MAet al.; Irwin/McGraw-Hill. 